\title{
In situ targeted activation of an anticancer agent using ultrasound- triggered release of composite droplets
}

\author{
Marine Bezagu, ${ }^{\mathrm{a}, \mathrm{c}}$ Jonathan Clarhaut, ${ }^{\mathrm{b}}$ Brigitte Renoux, ${ }^{\mathrm{b}}$ Fabrice Monti, ${ }^{\mathrm{c}}$ Mickael Tanter, ${ }^{\mathrm{d}}$ \\ Patrick Tabeling, ${ }^{\mathrm{c}, *}$ Janine Cossy, ${ }^{\mathrm{a}, *}$ Olivier Couture, ${ }^{\mathrm{d}}$ Sebastien Papot, ${ }^{\mathrm{b}, *}$ and Stellios Arseniyadis ${ }^{\mathrm{a}, \mathrm{e}, *}$ \\ ${ }^{a}$ Laboratoire de Chimie Organique, Institute of Chemistry, Biology and Innovation (CBI) - ESPCI Paris/CNRS (UMR8231)/PSL \\ Research University, 10 rue Vauquelin, 75231 Paris Cedex 05, France. \\ ${ }^{\mathrm{b}}$ Université de Poitiers, UMR-CNRS 7285, Institut de Chimie des Milieux et des Matériaux de Poitiers, groupe « Systèmes \\ Moléculaires Programmés », 4 rue Michel Brunet, TSA 51106, 86073 Poitiers, France. \\ ${ }^{c}$ Laboratoire de Microfluidique, MEMS et Nanostructures, ESPCI Paris, CNRS (UMR Gulliver 7083), 10 rue Vauquelin, 75231 \\ Paris Cedex 05, France. \\ ${ }^{\mathrm{d}}$ Institut Langevin, ESPCI Paris, CNRS (UMR 7587), INSERM (U979). 1 rue Jussieu, 75005 Paris, France. \\ ${ }^{\mathrm{e}}$ Queen Mary University of London, School of Biological and Chemical Sciences, Mile End Road, London, E1 4NS, UK.
}

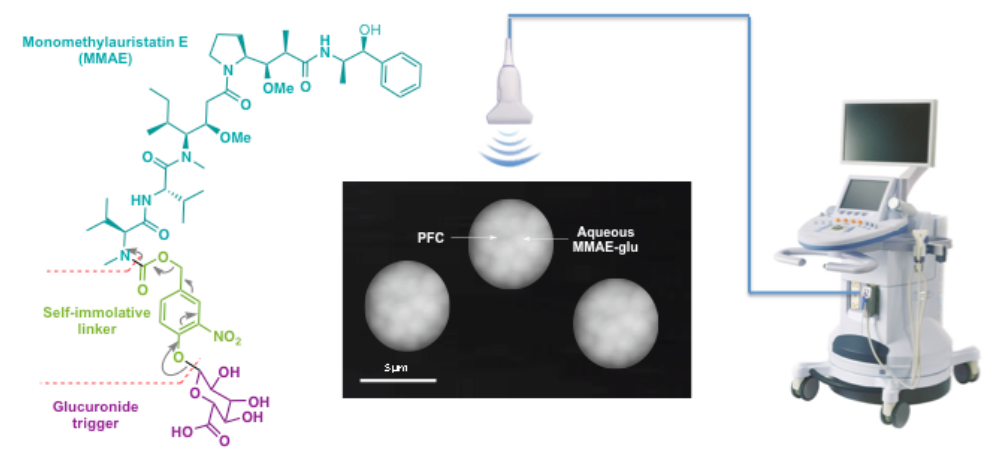

ABSTRACT: The efficiency of a drug is usually highly dependent on the way it is administered or delivered. As such, targeted-therapy, which requires conceiving drug-delivery vehicles that will change their state from a relatively stable structure with a very slow leak-rate to an unstable structure with a fast release, clearly improves the pharmacokinetics, the absorption, the distribution, the metabolism and the therapeutic index of a given drug. In this context, we have developed a particularly effective double stimuli-responsive drug-delivery method allowing an ultrasoundinduced release of a monomethylauristatin E-glucuronide prodrug and its subsequent activation by a $\beta$-glucuronidase. This led to an increase of cytotoxicity of about $80 \%$ on cancer cells.

\section{Introduction}

The development of 'smart' carriers programmed for the localized delivery of highly cytotoxic drugs in response to extracorporeal physical stimuli offers promises for cancer chemotherapy [1-3]. Such systems are indeed designed to release most, if not all, the administered drug dose directly at the tumor site thereby avoiding any undesired toxicity in healthy tissues. To date, several stimuli-triggered release methods have been reported in the literature; these include ultrasound-activated structures [4,5], especially microbubbles [6-10] or thermosensitive liposomes [11-16]. Considering that ultrasound benefit from their high spatial (millimeter) and temporal (microsecond) resolution, their depth of penetration (up to $10 \mathrm{~cm}$ ) and their ubiquity in hospitals worldwide [17], they represent a method of choice for achieving optimal spatial- and temporal-controlled release in the context of drug delivery. Within this framework, we previously demonstrated that ultrasound-sensitive perfluorocarbon (PFC) composite droplets injected systemically could transport large payload of fluorescein and deliver their content in vivo using a clinical ultrasound scanner with a millimetric resolution [18,19]. This strategy was also used by Fabiilli and co-workers to carry and release chlorambucil, a lipophilic chemotherapeutic [20], and thrombin, a serine protease used in the treatment of pseudoan- eurysms [21]. More recently, Fabiilli et al. also reported the biodistribution of such composite droplets of perfluorocarbon ( $6 \mu \mathrm{m}$ or less in diameter) in rats [22]. While this technology appeared very useful for internal tattooing of tissues, the slow leakage of the encapsulated compound observed from uninduced droplets appeared as a potential drawback to envision the adaptation of this concept to the delivery of highly toxic anticancer agents. Since the problem of unspecific release is inherent to all the carriers developed so far [23], we herein propose to evaluate a new generation of ultrasound-sensitive drug delivery systems based on the encapsulation, within ultrasound-sensitive perfluorocarbon composite droplets, of a none/less active drug precursor instead of the parent drug. Indeed, by specifically manufacturing the active agent within the ultrasound focus, not only would we be able to exclusively limit its activity within the zone delineated on the imaging scanner, but we would also limit any undesired side effects, which could occur downstream.

In order to evaluate the feasibility of this new drug delivery concept, we previously demonstrated that we were able to trigger a spontaneous reaction with a high spatial and temporal control [24]. A first proof of concept was achieved by promoting a $\mathrm{Cu}$-free click reaction using a single ultrasound pulse specifically within the focus of a transducer ( $0.6 \mathrm{~mm}$ in width) and within a time frame of less than $3 \mathrm{~ms}$. Since these acoustic 
pulses were produced by a clinical ultrasonic scanner, the generation of a chemical reaction deep into the tissue could clearly be envisioned. We therefore decided to test this hypothesis by investigating the in vitro ultrasound-induced activation of a glucuronide anticancer prodrug on cancer cells. We report here the results of our endeavor.

\section{Results and discussion}

A glucuronide prodrug of monomethylauristatin $\mathrm{E}$ (MMAE-glu) [25-32] was chosen to perform this study $[33,34]$. Indeed, MMAE is a potent antimitotic agent currently employed in human for the treatment of lymphomas under the form of the brentuximab vedotin, an antibody-drug conjugate that reached the market in 2011 [35]. In contrast, the free MMAE cannot be used clinically because of its excessive toxicity towards non-malignant tissues. Under such circumstances, encapsulation of MMAE-glu instead of MMAE brings several advantages. Indeed, as the prodrug is much more hydrophilic than the parent drug, it is therefore a much better candidate for encapsulation in aqueous composite droplets. In addition, the glucuronide prodrug is $40-100$ fold less toxic than the parent drug, which will largely reduce any nonselective toxicity caused by potential leakage of the composite droplets outside the area of interest and therefore limit potential damages of surrounding healthy tissues. Moreover, since the $\beta$-glucuronidase is over-expressed in the microenvironment of almost all solid tumors [26,36,37], ultrasound-triggered release of the prodrug in the vicinity of the tumor mass should be followed by the local enzyme-mediated release of the active drug via the self-immolative mechanism illustrated in Figure $1[38,39]$.

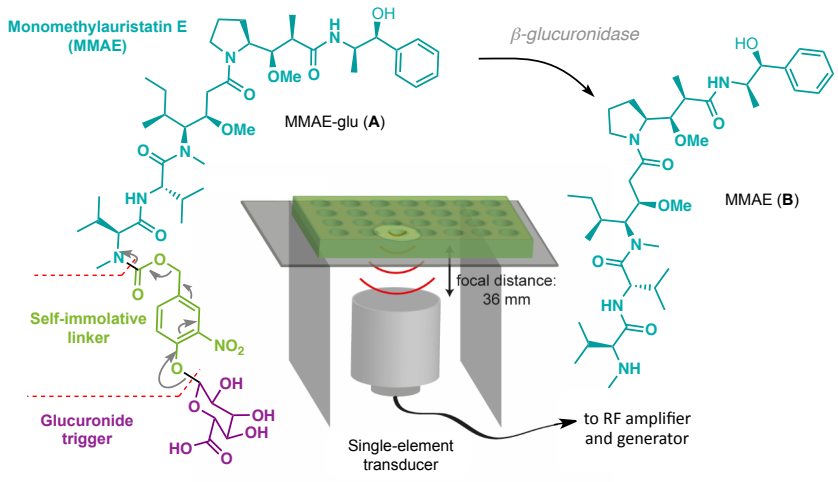

Fig. 1. Structure of MMAE glucuronide prodrug (MMAE-glu) and its self-immolative mechanism upon $\beta$-glucuronidase activation.

This strategy has proved its efficacy in numerous animal models over the years [29,37,40-45], as for example showed by Papot and co-workers in mice [30]. These prodrugs are rapidly eliminated by the kidneys, thus lowering their concentration in tumors. Nevertheless, the localized ultrasoundtriggered delivery of an encapsulated prodrug should also improve its therapeutic index by both enhancing its concentration in the vicinity of the targeted tissues and minimizing its metabolization.

We first encapsulated solutions of MMAE and glucuronylated MMAE (at a concentration of $0.1 \mathrm{mmol}^{-1}$ in a RPMI1640 cell culture) in the inner phase of composite drop- lets of perfluorocarbon. These droplets were produced with a microfluidic system composed of parallel step emulsifiers (see experimental protocols for more details). A specific microfluidic device could yield a monodisperse population of droplets with sizes varying between 3 and $8 \mu \mathrm{m}$. In the context of this study, the droplets had a mean diameter of $3.3 \mu \mathrm{m}$ with a standard deviation of $0.3 \mu \mathrm{m}$. Most importantly, these droplets are comparable in size and formulation to those previously exploited in-vivo by our group [18,19] and by the group of Fabiilli [20-22]. The in vitro study was carried out in 96-well plates specially designed to meet both the requirements of acoustic wave propagation and the biological needs (sterile medium, optical lecture of cell proliferation). Hence, the regular thick bottom of the plates was replaced by a thin Mylarmembrane. Those plates were immersed in a water bath thermo-stated at $37^{\circ} \mathrm{C}$, in which a single-element transducer was fixed at a distance from the plate corresponding to the focal distance of the transducer $(36 \mathrm{~mm})$. Four acoustic pulses were focused in each well ( 5 cycles $\approx 11 \mathrm{MPa} \mathrm{PNP}, 1.5 \mathrm{MHz}$, each pulse approximately one second apart).

Two different protocols were implemented (see experimental protocols for more details). In the first one, referred to as the "contact" protocol, KB cancer cells were grown in the wells for a $24 \mathrm{~h}$ period, before the droplets were added. Ultrasound pulses were then focused in the well in order to partially release the encapsulated compounds and the resulting suspension of unreleased droplets was left in contact with the cells and incubated at $37^{\circ} \mathrm{C}$ for $72 \mathrm{~h}$. The measurement of cell viability was then obtained through a XTT colorimetric assay. In the second protocol, referred to as the "transfer" protocol, a suspension of composite droplets was poured in an empty well. Part of the droplet's population was released using the same acoustic protocol. Only the droplets' supernatant, and therefore the ultrasound-released compounds, were transferred to the cancer cells, while the residual unreleased droplets were eliminated. After an incubation period of $72 \mathrm{~h}$ at $37{ }^{\circ} \mathrm{C}$, cell proliferation was evaluated using the same XTT assay.

In order to rule out false positives, a number of control experiments were first carried out. It was indeed essential to assess the effect of the ultrasound pulses, the droplets' composition and the ultrasound-mediated vaporization protocol on the viability of $\mathrm{KB}$ cells. In other terms, we needed to ensure that the cytotoxic effect we would be measuring would be solely resulting from the local release of the prodrug and its specific activation. Interestingly, ultrasound exposure showed no significant alteration of the cell viability in all cases. Similarly, prolonged exposure ("contact" protocol, $72 \mathrm{~h}$ exposure) to a high concentration $(0.5 \% \mathrm{v} / \mathrm{v})$ of droplets containing only cell culture medium (CM) did not lead to significant change in mortality. Finally, the combination of droplets and ultrasound to induce local PFC vaporization appeared to be harmless as well (Figure 2).

Considering the results of the control experiments, we pursued our investigation further by studying the in situ release and activation of both monomethylauristatin E (MMAE) and its glucuronide analogue (MMAE-glu). To do so, we evaluated composite droplets containing MMAE and MMAE-glu, both prepared at the same initial inner concentration of $0.1 \mathrm{mM}$.

A suspension of droplets at $0.01 \% \mathrm{v} / \mathrm{v}$ was used in the course of this study. Regarding the in situ activation of MMAE-glu by the $\beta$-glucuronidase ( $\beta$-glu), Legigan et al. [30] showed that complete conversion of the prodrug intro MMAE in the presence of $\beta$-glucuronidase was almost completely 


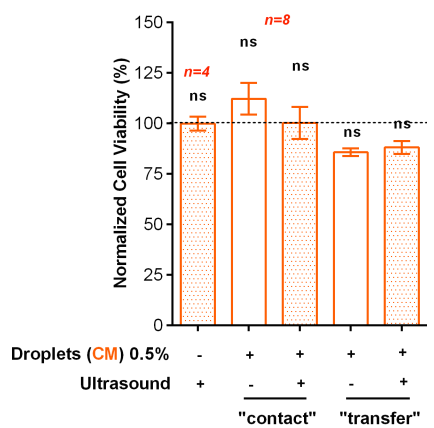

Fig. 2. Control experiments ("contact" protocol), using droplets containing pure cell culture medium $(\mathrm{CM})$. When relevant, 4 ultrasound pulses were focused on the corresponding wells (5 cycles, $11 \mathrm{MPa}$ PNP, $1.5 \mathrm{MHz}$ ). Cell viability was measured after a $72 \mathrm{~h}$ incubation period at $37{ }^{\circ} \mathrm{C}$ through a XTT colorimetric assay. All cell viability measurements were normalized by the viability obtained for non-treated cells. ns: no statistical difference. Experiment repeated $n$ times.

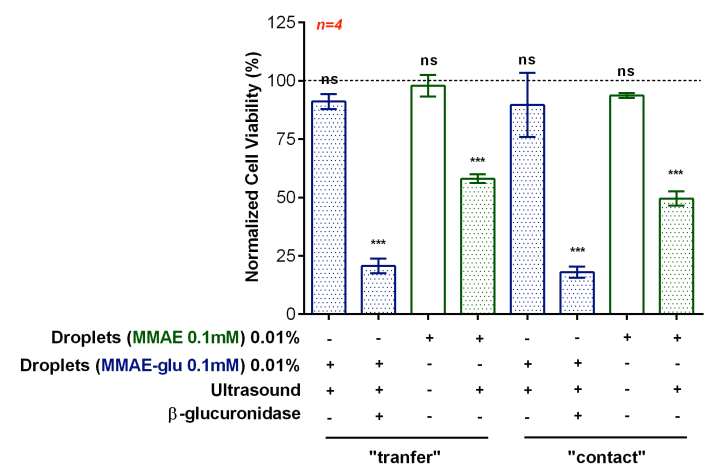

Fig. 3. In situ activation experiments ("transfer" and "contact" protocols), using droplets containing MMAE and MMAE-glu respectively. When relevant, $\beta$-glucuronidase was added (40u/well) and/or 4 ultrasound pulses were focused on the corresponding wells (5 cycles, $11 \mathrm{MPa}$ PNP, $1.5 \mathrm{MHz}$ ). Cell viability was measured after a $72 \mathrm{~h}$ incubation period at $37{ }^{\circ} \mathrm{C}$ through a XTT colorimetric assay. All cell viability measurements were normalized by the viability obtained for non-treated cells. Statistical significance was determined by ANOVA test and Dunnet post-test. ${ }^{*} \mathrm{p}<0.05,{ }^{* *} \mathrm{p}<0.01, * * * \mathrm{p}<0.001$, ns: no statistical difference. Experiment repeated 4 times $(n=4)$.

achieved within the first 10 minutes after contact. We were then able to compare the normalized cell viability of cells exposed to the ultrasound-released content of MMAE-glu droplets to the viability of cells exposed to both $\beta$-glucuronidase and ultrasound-released MMAE-glu. As a general trend, no statistically significant alteration of the cell viability was observed in the first case, while the exposure to both enzyme and released MMAE-glu led to a drop in viability of about $80 \%$ as a result of the in situ $\beta$-glucuronidasecatalyzed release of MMAE (Figure 3). A similar behavior was also observed in the case of a prolonged residence time of the droplets in the culture wells ("contact" protocol, $72 \mathrm{~h}$ exposure). Interestingly, however, the ultrasound-release of the encapsulated parent drug led to a smaller antiproliferative effect, which is most probably due to a slight difference in the

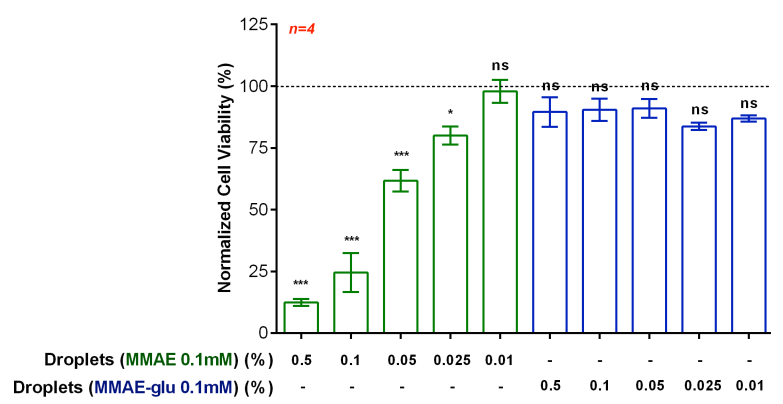

Fig. 4. Study of the droplets' stability and of the leakage phenomenon using the "transfer" protocol. Droplets containing either MMAE or MMAE-glu were used. Cell viability was measured after a $72 \mathrm{~h}$ incubation period at $37{ }^{\circ} \mathrm{C}$ through a XTT colorimetric assay without adding any $\beta$-glucuronidase in the wells. All cell viability measurements were normalized by the viability obtained for non-treated cells. Statistical significance was determined by ANOVA test and Dunnet post-test. $* \mathrm{p}<0.05, * * \mathrm{p}<0.01, * * * \mathrm{p}<0.001$, ns: no statistical difference. Experi-ment repeated 4 times $(n=4)$.

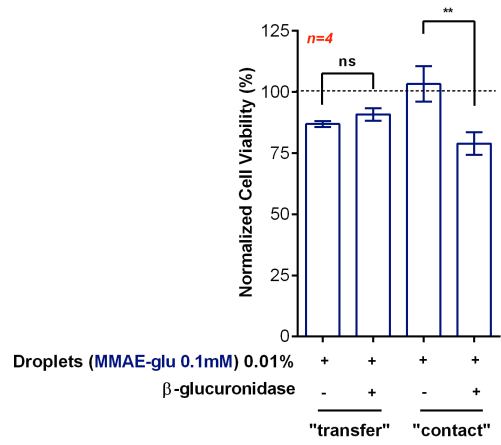

Fig. 5. Study of the droplets' stability and of the leakage phenomenon using the "transfer" protocol. Droplets containing either MMAE or MMAE-glu were used. Cell viability was measured after a $72 \mathrm{~h}$ incubation period at $37{ }^{\circ} \mathrm{C}$ through a XTT colorimetric assay without adding any $\beta$-glucuronidase in the wells. All cell viability measure-ments were normalized by the viability obtained for non-treated cells. Statistical significance was determined by ANOVA test and Dunnet post-test. $* \mathrm{p}<0.05, * * \mathrm{p}<0.01, * * * \mathrm{p}<0.001$, ns: no statistical differ-ence. Experiment repeated 4 times $(n=4)$.

respective MMAE and MMAE-glu droplets' formulation; the prodrug being more hydrophilic than the parent drug.

Concerned by the potential damage that could cause passive leakage of a highly toxic drug such as MMAE, we next evaluated the impact of the leakage of MMAE-glu containing droplets. To this end, KB cells were incubated for $72 \mathrm{~h}$ with increased concentrations of droplets containing either MMAE or MMAE-glu ("transfer" protocol) without adding any $\beta$-glucuronidase. As shown in Figure 4, increasing the concentration of MMAE-containing droplets dramatically affected the cell viability. In sharp contrast, MMAE-glu droplets exhibited no significant toxicity until the highest tested concentration of $0.5 \% \mathrm{v} / \mathrm{v}$ thus showcasing the benefits of encapsulating the prodrug instead of the parent drug. 
After demonstrating that MMAE-glu could be locally reactivated into MMAE upon ultrasound release and subsequent $\beta$-glucuronidase-mediated activation, and after confirming the limited impact of any residual leakage that could potentially stem from our prodrug-containing droplets, we brought additional evidence of the potential of our double-stimuli responsive strategy by showing the importance of the joint ultrasound and enzymatic activation. Remarkably, prolonged exposure of the cells to the MMAE-glu and MMAEcontaining droplets ("contact" protocol, $72 \mathrm{~h}$ exposure) showed no statistically relevant drop in cell viability.

We also compared the viability of the cells treated with MMAE-glu-containing droplets in the presence and in the absence of $\beta$-glucuronidase without using any ultrasound. Interestingly, in the case of short-term leakage ("transfer" protocol), no significant change in cell viability was observed with both droplets in the presence or in the absence of enzyme in the culture medium (Figure 5). After a prolonged contact time of the MMAE-glu containing droplets with the cells ("contact" protocol, $72 \mathrm{~h}$ exposure), only a minute increase in cell mortality was detected, which showcased both the very good retention of the encapsulated compounds into the droplets and the absolute necessity of the $\beta$-glucuronidase to trigger the activation of the drug.

\section{Conclusion}

In conclusion, we managed to encapsulate an anti-cancer agent, monomethylauristatin E (MMAE), and its glucuronide prodrug, MMAE-glu, into composite droplets of perfluorohexane. A good retention of those compounds into the droplets was observed over three days. Moreover, cytotoxic activity of the parent drug MMAE was successfully restored by ultrasound-release of MMAE-glu droplets in the presence of $\beta$-glucuronidase with an $80 \%$ increase in cell mortality compared to the ultrasound release in the absence of the enzymatic partner. These in vitro results on KB cancer cells clearly validate our proof of concept. In the case of an enzymatic activation such as the one provided in this study, as the enzyme is specific of the extra-tumoral medium, the in vivo activation should only occurs on the tumor site. This in situ chemistry strategy could potentially be extended to other example of prodrug/enzyme couples as well as to other kind of chemically activatable systems [46].

\section{Experimental protocols}

\subsection{Composite droplet preparation.}

The composite droplets were fabricated in two steps. First, a primary nano-emulsion was obtained by using a sonic tip (Branson, $20 \mathrm{kHz}, 70 \%$ of maximum amplitude, 8 cycles of $5 \mathrm{sec}$ ), with the fluids immersed in ice water. This nanoemulsion consists of an aqueous solution containing respectively MMAE $(0.1 \mathrm{mM})$ and MMAE-glu $(0.1 \mathrm{mM})$ in cell culture medium dispersed in perfluorohexane (FC-72, 3M) and stabilized by a fluorinated surfactant (PEG-di-Krytox, RAN Biotechnologies, $3 \% \mathrm{w} / \mathrm{v}$ in perfluorocarbon phase). The size of these nanoemulsions was evaluated on a Malvern Zetasizer Nano ZS. Typical diameter was found to be between 300 and $500 \mathrm{~nm}$. The primary nano-emulsion was then encapsulated as micron-size droplets in a PBS solution (1X from 10X commercial solution), using a microfluidic device based on a par- allelized system of step emulsifiers. The microfluidic system was made of polydimethylsiloxane. The droplets were stabilized by Pluronic-68® (polyoxyethylene-polyoxypropylene block copolymer, Sigma-Aldrich, St. Louis, MO, 3\% w/v). The droplets were collected. Supernatant from the production step was removed and replaced by another PBS solution with various additives (Pluronic-68®, 3\% w/v, Penicillin/Streptomycin 2X, Gentamycin 2X, Kanamycin 2X) to prevent any bacterial development. Droplets were then stored at $4{ }^{\circ} \mathrm{C}$ and used within $48 \mathrm{~h}$.

\subsection{Cell culture}

KB (human oral squamous carcinoma) cells were grown in RPMI 1640 (Invitrogen) supplemented by 10\% fetal bovine serum and $1 \%$ Penicillin/Streptomycin (Lonza) in a humidified incubator at $37{ }^{\circ} \mathrm{C}$ and $5 \% \mathrm{CO} 2$. KB cells were purchased on ATCC (CCL-17) and used at early passages.

\subsection{Ultrasound set-up}

An ultrasound transducer $(2.25 \mathrm{MHz}, 38 \mathrm{~mm}$ focal length, $\mathrm{f} / \mathrm{d}=1$, Imasonics, Voray-sur-l'Ognon, France) was fixed in a 3D-printed plastic setup, which allowed a 96-well plate immersed in a water bath thermostated at $37^{\circ} \mathrm{C}$ to be moved through the acoustic focus (Figure 2a). The transducer was powered with a $40 \mathrm{~dB}$ RF amplifier (TOMCO, Stepney, Australia) driven by an arbitrary waveform generator (Tektronix, Beaverton, Oregon). Four acoustic pulses (5 cycles, $11 \mathrm{MPa}$ PNP, $1.5 \mathrm{MHz}$ ) were triggered after aligning the target-well with the acoustic focus. The 96 well-plates were bottomless plates under which a Mylar membrane was glued under sterile conditions to provide an optically and acoustically transparent window.

\subsection{Contact protocol}

$\mathrm{KB}$ cells were seeded and grown for $24 \mathrm{~h}$ in poly-L-lysinecoated thin-bottom 96-well plates. After this first incubation period at $37{ }^{\circ} \mathrm{C}, 70 \mu \mathrm{L}$ of a $0.01 \% \mathrm{v} / \mathrm{v}$ suspension of droplets in cell culture medium was placed in each well, on the cells. When relevant, 4 ultrasound pulses were focused on the corresponding wells, followed when necessary by a solution of $\beta$-glucuronidase (E. coli, 40u/well). The plates were then incubated at $37^{\circ} \mathrm{C}$ for $72 \mathrm{~h}$, before XTT assay was performed to evaluate cell proliferation.

\subsection{Transfer protocol}

KB cells were seeded and grown for $24 \mathrm{~h}$ in regular 96well plates. After this first incubation period at $37^{\circ} \mathrm{C}, 90 \mu \mathrm{L}$ of a $0.01 \% \mathrm{v} / \mathrm{v}$ suspension of droplets in cell culture medium was placed in thin-bottom 96-well plates. When relevant, 4 ultrasound pulses were focused on the corresponding wells (5 cycles, $11 \mathrm{MPa}$ PNP, 1.5 MHz). The content of each well was centrifuged, and the non-released droplets were eliminated. Supernatant containing the ultrasound-released compounds $(70 \mu \mathrm{L})$ was then transferred on the KB cells in the regular 96-well plates, followed when necessary by a solution of $\beta$-glucuronidase (E. coli, $40 \mathrm{u} /$ well). The plates were then incubated at $37{ }^{\circ} \mathrm{C}$ for $72 \mathrm{~h}$, before XTT assay was performed to evaluate cell proliferation. 


\subsection{Cell viability measurements}

The cell viability kit II (XTT, Roche) was used to assess cell viability. This assay is based on the cleavage of XTT by metabolic active cells resulting in the production of an orange formazan dye quantified by spectrophotometry. Assays were carried out essentially as described by the manufacturer. Briefly, after the incubation specified in each protocol, $25 \mu \mathrm{L}$ of the XTT labeling mixture were added per well. Cells were further incubated for additional $4 \mathrm{~h}$ at $37{ }^{\circ} \mathrm{C}$ before determination of the absorbance at $490 \mathrm{~nm}$ (Absorbance was read using a multimode microplate reader (Mithras LB940, Berthold Technologies). Experiments were performed $n$ times $(4<n<8)$ per condition. Statistical significance was determined by ANOVA test and Dunnet post-test. ${ }^{*} \mathrm{p}<0.05, * * \mathrm{p}<0.01, * * * \mathrm{p}<0.001$, ns: no statistical difference.

\section{Acknowledgment}

This work was supported by the LABEX WIFI (Laboratory of Excellence ANR-10-LABX-24) within the French Program "Investments for the Future" under the reference ANR-10-IDEX0001-02 PSL*. MB and $\mathrm{JCl}$ would like to thank the Institut Pierre-Gilles de Gennes pour la microfluidique, L'Oréal Unesco For Women in Science and Sport and Collection for funding.

\section{Appendix A. Supplementary data}

Supplementary data related to this article can be found at

\section{Corresponding Authors}

*E-mail: janine.cossy@espci.fr.

*E-mail: sebastien.papot@univ-poitiers.fr

*E-mail: s.arseniyadis@qmul.ac.uk

\section{References}

[1] S. Mura, J. Nicolas, P. Couvreur, Stimuli-responsive nanocarriers for drug delivery, Nat. Mater. 12 (2013) 991-1103.

[2] M. J. Webber, E. A. Appel, E. W. Meijer, R. Langer, Supramolecular biomaterials, Nat. Mater. 15 (2016) 13-26.

[3] Y. Min, J. M. Caster, M. J. Eblan, A. Z. Wang, Clinical translation of nanomedicine, Chem. Rev. 15 (2016) 11147-11190.

[4] K. W. Ferrara, Driving delivery vehicles with ultrasound, Adv. Drug Deliv. Rev. 60 (2008) 1097-1102.

[5] O. Couture, J. Foley, N. F. Kassell, B. Larrat, J.-F. Aubry, Review of ultrasound mediated drug delivery for cancer treatment: updates from pre-clinical studies, Transl. Cancer Res. 3 (2014) 494-511.

[6] S. Hernot, A. L. Klibanov, Microbubbles in ultrasoundtriggered drug and gene delivery, Adv. Drug Deliv. Rev. 60 (2008) 1153-1166.

[7] I. Lentacker, S. C. De Smedt, N. N. Sander, Drug loaded microbubble design for ultrasound triggered delivery, Soft Matter 5 (2009) 2161-2170.

[8] B. Geers, H. Dewitte, S. C. De Smedt, I. Lentacker, Crucial factors and emerging concepts in ultrasound-triggered drug delivery, J. Control. Release 164 (2012) 248-255.

[9] S. Tinkov, C. Coester, S. Serba, N. A. Geis, H. A. Katus, G. Winter, R. Bekeredjian, New doxorubicin-loaded phospholipid microbubbles for targeted tumor therapy: in-vivo characterization, J. Control. Release 148 (2010) 368-372.
[10] S. Ibsen, C. E. Schutt, S. Esener, Microbubble-mediated ultrasound therapy: a review of its potential in cancer treatment, Drug Des. Dev. Ther. 7 (2013) 375-388.

[11] J. N. Weinstein, R. L. Magin, M. B. Yatvin, D. S. Zaharko, Liposomes and local hyperthermia: Selective delivery of methotrexate to heated tumors, Science 204 (1979) 188-191.

[12] A. Schroeder, J. Kost, Y. Barenholz, Ultrasound, liposomes, and drug delivery: principles for using ultrasound to control the release of drugs from liposomes, Chem. Phys. Lipids 162 (2009) 1-16.

[13] J. A. Mackay, A. Chilkoti, Temperature sensitive peptides: engineering hyperthermia-directed therapeutics, Int. J. Hyperthermia 24 (2008) 483-500.

[14] Z. S. Al-Ahmady, W. T. Al Jamal, J. V. Bossche, T. T. Bui, A. F. Drake, A. J. Mason, K. Kostarelos, Lipid-peptide vesicle nanoscale hybrids for triggered drug release by mild hyperthermia in vitro and in vivo, ACS Nano 6 (2012) 9335-9346.

[15] C. Mannaris, E. Efthymiou, M.-E. Meyre, M. A. Averkiou, In vitro localized release of thermosensitive liposomes with ultrasound-induced hyperthermia, Ultrasound in Med. \& Biol. 39 (2013) 2011-2020.

[16] https://clinicaltrials.gov/ct2/show/NCT00826085

[17] S. Mitragotri, Healing sound: the use of ultrasound in drug delivery and other therapeutic applications, Nat. Rev. Drug Discov. 4 (2005) 255-260.

[18] O. Couture, M. Faivre, N. Pannacci, A. Babataheri, V. Servois, P. Tabeling, M. Tanter, Ultrasound internal tattooing, Med. Phys. 38 (2011) 1116-1123.

[19] O. Couture, A. Urban, A. Bretagne, L. Martinez, M. Tanter, P. Tabeling, In vivo targeted delivery of large payloads with an ultrasound clinical scanner, Med. Phys. 39 (2012) 5229-5237.

[20] M. L. Fabiilli, K. J. Haworth, I. E. Sebastian, O. D. Kripfgans, P. L. Carson, J. B. Fowlkes, Delivery of chlorambucil using an acoustically-triggered perfluoropentane emulsion, Ultrasound Med. Biol. 36 (2010) 1364-1375.

[21] M. L. Fabiilli, J. A. Lee, O. D. Kripfgans, P. L. Carson, J. B. Fowlkes, Delivery of Water-Soluble Drugs Using Acoustically Triggered Perfluorocarbon Double Emulsions, Pharm. Res. 27 (2010) 2753-2765.

[22] M. L. Fabiilli, M. R. Piert, R. A. Koeppe, P. S. Sherman, C. A. Quesada, O. D. Kripfgans, Assessment of the biodistribution of an [18F]FDG-loaded perfluorocarbon double emulsion using dynamic micro-PET in rats, Contrast Media Mol. Imaging 8 (2013) 366-374.

[23] Y. V. Kalinin, A. Murali, D. H. Gracias, Chemistry with spatial control using particles and streams, RSC Adv. 2 (2012) 97079726.

[24] M. Bezagu, C. Errico, V. Chaulot-Talmon, F. Monti, M. Tanter, P. Tabeling, J. Cossy, S. Arseniyadis, O. Couture, High spatiotemporal control of spontaneous reactions using ultrasoundtriggered composite droplets, J. Am. Chem. Soc. 136 (2014) 7205-7208.

[25] L. F. Tietze, R. Seele, B. Leiting, T. Krach, Stereoselective synthesis of (1-alkoxyalkyl) $\alpha$ - and $\beta$-D-glucopyranosiduronates (acetal-glucopyranosiduronates): a new approach to specific cytostatics for the treatment of cancer, Carbohydr. Res. 180 (1988) 253-262.

[26] K. Bosslet, R. Straub, M. Blumrich, J. Czech, M. Gerken, B. Sperker, H. K. Kroemer, J.-P. Gesson, M. Koch, C. Monneret, Elucidation of the mechanism enabling tumor selective prodrug monotherapy, Cancer Res. 58 (1998) 1195-1201.

[27] D. Weyel, H.-M. Sedlacek, R. Müller, S. Brüsselbach, Secreted human bold $\beta$-glucuronidase: a novel tool for gene-directed enzyme prodrug therapy, Gene Ther. 7 (2000) 224-231.

[28] T. Mürdter, G. Friedel, J. T. Backman, M. McClellan, M. Schick, M. Gerken, K. Bosslet, P. Fritz, H. Toomes, H. K. Kroemer, B. J. Sperker, Dose optimization of a doxorubicin prodrug (HMR 1826) in isolated perfused human lungs: low tumor $\mathrm{pH}$ promotes prodrug activation by $\beta$-glucuronidase, Pharm. Exp. Ther. 301 (2002) 223-228. 
[29] I. Tranoy-Opalinski, T. Legigan, R. Barat, J. Clarhaut, M. Thomas, B. Renoux, S. Papot, $\beta$-Glucuronidase-responsive prodrugs for selective cancer chemotherapy: An update, Eur. J. Med. Chem. 74 (2014) 302-313.

[30] T. Legigan, J. Clarhaut, B. Renoux, I. Tranoy-Opalinski, A. Monvoisin, C. Jayle, J. Alsarraf, M. Thomas, S. Papot, Synthesis and biological evaluations of a monomethylauristatin E glucuronide prodrug for selective cancer chemotherapy, Eur. J. Med. Chem. 67 (2013) 75-80.

[31] A. Balbous, B. Renoux, U. Cortes, S. Milin, K. Guilloteau, T. Legigan, P. Rivet, O. Boissonnade, S. Martin, C. Tripiana, M. Wager, R.-J. Bensadoun, S. Papot, L. Karayan-Tapon, Selective release of a cyclopamine glucuronide prodrug toward stem-like cancer cell inhibition in glioblastoma, Mol. Cancer Ther. 13 (2014) 2159-2169.

[32] S. Bensalma, C. Chadeneau, T. Legigan, B. Renoux, A. Gaillard, M. De Boisvilliers, C. Pinet-Charvet, S. Papot, J.-M. Muller, Evaluation of cytotoxic properties of a cyclopamine glucuronide prodrug in rat glioblastoma cells and tumors, J. Mol. Neurosci. 55 (2015) 51-61.

[33] J. Rautio, H. Kumpulainen, T. Heimbach, R. Oliyai, D. Oh, T. Järvinen, J. Savolainen, Prodrugs: design and clinical applications, Nat. Rev. Drug Discov. 7 (2008) 255-270.

[34] V. Abet, F. Filace, J. Recio, J. Alvarez-Builla, C. Burgos, Prodrug approach: An overview of recent cases, Eur. J. Med. Chem. 127 (2017) 810-827.

[35] S. O. Doronina, B. E. Toki, M. Y. Torgov, B. A. Mendelsohn, C. G. Cerveny, D. F. Chace, R. L. DeBlanc, R. P. Gearing, T. D. Bovee, C. B. Siegall, J. A. Francisco, A. F. Wahl, D. L. Meyer, P. D. Senter, Development of potent monoclonal antibody auristatin conjugates for cancer therapy, Nat. Biotechnol. 21 (2003) 778-784.

[36] T. A. Connors, M. E. Whisson, Cure of mice bearing advanced plasma cell tumours with aniline mustard: the relationship between glucuronidase activity and tumour sensitivity, Nature 210 (1966) 866-867.
[37] N. Albin, L. Massaad, C. Toussaint, M.-C. Mathieu, J. Morizet, O. Parise, A. Gouyette, G. G. Chabot, Main drug-metabolizing enzyme systems in human breast tumors and peritumoral tissues, Cancer Res. 53 (1993) 3541-3546.

[38] S. Papot, I. Tranoy, F. Tillequin, J.-C. Florent, J.-P.Gesson, Design of selectively activated anticancer prodrugs: elimination and cyclization strategies, Curr. Med. Chem. Anticancer Agents 2 (2002) 155-185.

[39] I. Tranoy-Opalinski, A. Fernandes, M. Thomas, J.-P. Gesson, S. Papot, Design of self-immolative linkers for tumour-activated prodrug therapy, Anticancer Agents Med. Chem. 8 (2008) 618637.

[40] A. V. Stachulski, X. Meng, Glucuronides from metabolites to medicines: a survey of the in vivo generation, chemical synthesis and properties of glucuronides, Nat. Prod. Rep. 30 (2013) 806-848.

[41] W. H. Fishman, A. J. Anlyan, Comparison of the $\beta$-glucuronidase activity of normal tumor and lymph node tissues of surgical patients, Science 106 (1947) 66-67.

[42] E. Boyland, D. M. Wallace, D. C. Williams, The activity of the enzymes sulphatase and $\beta$-glucuronidase in the urine, serum and bladder tissue, Br. J. Cancer 9 (1955) 62-79.

[43] K. Bosslet, J. Czech, D. Hoffmann, Tumor-selective prodrug activation by fusion protein-mediated catalysis, Cancer Res. 54 (1994) 2151-2159.

[44] C. Nygren, H. von Holst, J. E. Månsson, P. Fredman, Increased activity of lysosomal glycohydrolases in glioma tissue and surrounding areas from human brain, Acta Neurochir. 139 (1997) 146-150.

[45] B. Sperker, U. Werner, T. E. Mürdter, C. Tekkaya, P. Fritz, R. Wacke, U. Adam, M. Gerken, B. Drewelow, H. K. Kroemer, Expression and function of $\beta$-glucuronidase in pancreatic cancer: potential role in drug targeting, Naunyn-Schmiedebergs Arch. Pharmacol. 362 (2000) 110-115.

[46] R. van Brakel, R. C. M. Vulders, R. J. Bokdam, H. Grüll, M. S. A. Robillard, A Doxorubicin prodrug activated by the staudinger reaction, Bioconjug. Chem. 19 (2008) 714-718. 